\title{
ORGANIZAÇ̃̃O E ESTRUTURAÇÃO DO SETOR DE TRIAGEM DE PACIENTES EM CLÍNICA ODONTOLÓGICA UNIVERSITÁRIA
}

\section{ORGANIZATION AND STRUCTURING OF THE PATIENT TRIAGE SECTOR IN A UNIVERSITY DENTAL CLINIC}

\author{
ORCID: https://orcid.org/0000-0003-2384-6538 \\ Ana Cláudia Dalmolin** \\ ORCID: https://orcid.org/0000-0002-6824-7312 \\ Lauro Taques Neto*** \\ ORCID: https://orcid.org/0000-0001-5686-9475 \\ Mariane Aparecida Savi Sanson**** \\ ORCID: https://orcid.org/0000-0003-2197-7008 \\ Leomar Emanuel Almeida Mecca***** \\ ORCID: https://orcid.org/0000-0001-6187-7859 \\ Fábio André dos Santos****** \\ ORCID: https://orcid.org/0000-0003-0347-0270
}

\section{Resumo}

Data recebimento: 08/09/2020

Data de aceite: $15 / 03 / 2021$

Este estudo é um relato de experiência das ações iniciais do projeto de extensão intitulado "Estruturação do Setor de Triagem para as Clínicas Odontológicas da Universidade Estadual de Ponta Grossa". O processo de inovação Design Thinking foi utilizado para compreender os problemas do modelo de triagem existente, para posteriormente implementar soluções. As ações solucionadoras consistiram no estabelecimento de um fluxo de direcionamento dos pacientes desde a "porta de entrada" até a conclusão do tratamento, no uso do espaço físico da Recepção como local de referência para proporcionar informações e orientações, na centralização das ações do Setor de Triagem na Recepção, na organização da sala de arquivos e implementação de um prontuário odontológico único. Apesar do dinamismo do processo de Design Thinking e das ações iniciais realizadas, muitos problemas persistem, a implementação de mudanças demanda tempo e passa por dificuldades até que as melhorias alcançadas sejam consolidadas.

Palavras-chave: Saúde Pública; Saúde Bucal; Triagem; Resolução de Problemas.

\section{Abstract}

This study is a report of experiences from initial actions of the extension project entitled "Structuring the Triage Sector for the Dental Clinics at the State University of Ponta Grossa". The Design Thinking process was used to understand the problems of the existing triage model, to subsequently implement solutions. The solution actions consisted in establishing a direct flow of patients from the "gateway" to the end of treatment, by using the physical space of the Reception as a reference place to provide information and guidance, centralizing the actions of the Triage Sector in the Reception, organizing the file room and implementing a single dental record. Despite the dynamism of the Design Thinking process and the initial actions carried out, many problems persist, the implementation of changes takes time and goes through difficulties until the improvements achieved are consolidated.

Keywords: Public Health; Oral Health; Triage; Problem Solving.

* Aluna de Doutorado da Universidade Estadual de Ponta Grossa (UEPG), Paraná - Brasil. E-mail: danihuller@gmail.com

** Aluna de Doutorado da Universidade Estadual de Ponta Grossa (UEPG), Paraná - Brasil. E-mail: anaclaudiadalmolin@gmail.com

*** Aluno de Mestrado da Universidade Estadual de Ponta Grossa (UEPG), Paraná - Brasil. E-mail: lauroneto15@hotmail.com

**** Professora da Universidade Estadual de Ponta Grossa (UEPG), Paraná - Brasil. E-mail: sanson.mari@gmail.com

***** Aluno de Doutorado da Universidade Estadual de Ponta Grossa (UEPG), Paraná - Brasil. E-mail: leoo.mecca@gmail.com

****** Professor da Universidade Estadual de Ponta Grossa (UEPG), Paraná - Brasil. E-mail: fasantos@uepg.br 


\section{Introdução}

As doenças bucais constituem significativos problemas de saúde pública que impõem consideráveis encargos pessoais, sociais e econômicos (LISTL et al., 2015; PETERSEN, 2003). O seu impacto sobre indivíduos e comunidades resulta em dor, comprometimento da função, redução da qualidade de vida e restrição de atividades (PETERSEN, 2003). O impacto econômico global atribuído às doenças bucais foi estimado em US\$ 442 bilhões em 2010, incluindo custos diretos com tratamento e indiretos em termos de perda de produtividade devido ao absenteísmo no trabalho. A redução da produtividade foi atribuída à perda dentária (44\%), periodontite grave (38\%) e cárie não tratada (17\%) em dentes permanentes (LISTL et al., 2015).

Em vários países, o acesso aos serviços de saúde bucal ainda é limitado, consequentemente, muitos dentes acabam não recebendo tratamento ou são extraídos (PETERSEN, 2003). No Brasil, 11\% da população acima de 18 anos é totalmente desdentada, o que corresponde a um contingente de 16 milhões de pessoas (IBGE, 2015). Apenas a cobertura da assistência odontológica pública não consegue suprir a demanda da população brasileira (GONÇALVES; VERDI, 2007; GONÇALVES; VERDI, 2005). Segundo a Pesquisa Nacional de Saúde (2013), o atendimento odontológico no Brasil ocorre preponderantemente em consultório particular ou clínica privada, totalizando $74 \%$ dos atendimentos, enquanto as unidades básicas de saúde são responsáveis por apenas 20\% (IBGE, 2015).

O tratamento das doenças bucais é extremamente dispendioso (PETERSEN, 2003), sendo o atendimento particular inviável para a maioria das pessoas de baixa e média renda. Uma opção de assistência odontológica pública, procurada por um grande número de pessoas, são as clínicas de ensino vinculadas aos cursos de Odontologia das instituições de ensino superior (IES), as quais prestam atendimento básico e especializado, gratuito ou de baixo custo, por meio de profissionais ainda em formação (GONÇALVES; VERDI, 2007; GONÇALVES; VERDI, 2005).

Nesse contexto, as IES precisam de um Setor de Triagem eficiente para direcionar adequadamente os pacientes de acordo com a capacidade de atendimentos e procedimentos ofertados. A triagem no curso de Odontologia da Universidade Estadual de Ponta Grossa (UEPG) até 2016 era realizada de maneira isolada, sem um Setor de Triagem que estruturasse todas as etapas do processo. Os acadêmicos do $5^{\circ}$ ano, a partir de demanda espontânea, eram responsáveis pelo acolhimento, atendimento e encaminhamento dos pacientes para as demais clínicas de tratamento, estando longe de ser um modelo desejável.

A ineficiência de um Setor de Triagem ocasiona diversos problemas, os quais podem levar ao comprometimento da resolução das necessidades bucais do paciente. Percebendo que o processo de triagem do curso de Odontologia da UEPG apresentava defasagens, implementouse um projeto de extensão, que tem por objetivo principal estruturar o Setor de Triagem do curso, visando melhorar o atendimento à comunidade. Neste contexto, o presente trabalho relata as vivências a partir das ações iniciais do referido projeto. 


\section{Metodologia}

O presente trabalho é um relato de experiência a partir das atividades do projeto de extensão universitária intitulado "Estruturação do Setor de Triagem para as Clínicas Odontológicas da Universidade Estadual de Ponta Grossa", executadas no período de 09/2016 a 09/2018. A proposta do projeto foi idealizada considerando a necessidade de readequar e reestruturar o funcionamento da triagem odontológica na UEPG, visando melhorar o atendimento à comunidade. O projeto foi aprovado pela Pró-Reitoria de Extensão e Assuntos Culturais (número de identificação 11800.16.00481.03). As ações iniciais do projeto foram realizadas utilizando o processo de inovação denominado Design Thinking (VIANNA et al., 2012), o qual, através da colaboração dos envolvidos no processo, buscou entender os problemas do modelo de triagem então existente para posteriormente implementar soluções.

O passo inicial deu-se com a seleção dos integrantes do projeto. Além do professorcoordenador, fazem parte seis professores do departamento e cinco alunos do Programa de Pós-Graduação Stricto Sensu (mestrado e doutorado) em Odontologia. Da graduação, do primeiro ao quarto ano, 52 alunos se inscreveram para participar do projeto. Após o processo de seleção mediante entrevista, foram selecionados 20 alunos, aleatorizados em cinco equipes de trabalho. As equipes foram constituídas por um aluno de cada ano da graduação e um aluno de pós-graduação (coordenador da equipe). A operacionalização inicial do projeto consistiu em reuniões semanais de cada equipe e reuniões mensais com todos os acadêmicos e professores participantes.

O desafio inicial enfrentado foi compreender os problemas do modelo de triagem existente em todas as suas facetas, para posteriormente implementar soluções. Neste contexto, trabalhou-se com o processo de Design Thinking, o qual pode ser considerado um mecanismo de identificação de problemas, com abordagem centrada no humano, culminando na proposição de possibilidades de soluções, ou seja, inovação (DEITTE; OMARY, 2019; EBERHART et al., 2019; ROBERTS et al., 2016; IDEO, 2015). Baseia-se na ideia de que entender a experiência do usuário final, ou seja, das pessoas que diretamente vivenciam os impactos do serviço e para as quais se destina o serviço, auxilia a definir corretamente o problema e a desenvolver soluções adequadas, partindo da crença de que as pessoas que enfrentam o problema diariamente são as que possuem as ferramentas para a solução do mesmo (DEITTE; OMARY, 2019; EBERHART et al., 2019; DOCHERTY, 2017; ROBERTS et al., 2016; IDEO, 2015). Este método observa a realidade considerando o envolvimento entre diferentes atores-chave (pessoas envolvidas no processo) e ambientes (locais onde os atores atuam) (DEITTE; OMARY, 2019; DOCHERTY, 2017; ROBERTS et al., 2016; IDEO, 2015; VIANNA et al., 2012).

As etapas do processo de Design Thinking variam de acordo com o autor (ROBERTS et al., 2016). Neste projeto, o processo foi estruturado de acordo com as fases: 1. Fase de Imersão Preliminar; 2. Fase de Imersão em Profundidade; 3. Fase de Análise e Síntese; 4. Fase de Ideação; 5. Fase de Prototipação; e, por fim, 6. Fase de Implementação (VIANNA et al., 2012). 
A Fase de Imersão Preliminar visa o entendimento inicial do problema, identificando os atores-chave e ambientes onde atuam. Nesta fase, foram utilizadas as ferramentas: Pesquisa Exploratória e Pesquisa Desk. A Pesquisa Exploratória é uma pesquisa de campo, na qual a equipe observa e interage com as pessoas envolvidas no processo buscando entender o contexto do assunto trabalhado (EBERHART et al., 2019; VIANNA et al., 2012). A Pesquisa Desk consiste na busca de informações sobre o tema em fontes diversas, a fim de conhecer tendências da área estudada. O nome Desk origina-se de desktop, porque grande parte da pesquisa realizada atualmente tem como base referências disponibilizadas na internet (EBERHART et al., 2019; FERREIRA et al., 2015; VIANNA et al., 2012).

A Fase de Imersão em Profundidade destina-se à identificação das necessidades, perspectivas e anseios dos atores-chave, através de um mergulho a fundo no assunto trabalhado (IDEO, 2015; VIANNA et al., 2012). Nesta fase, foram realizadas entrevistas com os atores identificados na fase anterior (EBERHART et al., 2019; IDEO, 2015; VIANNA et al., 2012). As entrevistas foram elaboradas em formato de questionário semiestruturado, submetidas à Plataforma Brasil como projeto de pesquisa e sua aprovação deu-se pelo Comitê de Ética em Pesquisa envolvendo Seres Humanos da UEPG (CEP/UEPG - parecer de aprovação: 2.788.307).

A Fase de Análise e Síntese objetiva organizar dados qualitativos com certo grau de sistematização, permitindo identificar e interpretar problemas de difícil percepção (LISLE; MERENDA; GABBARD, 2019). Nesta fase, foram utilizadas as ferramentas: Cartões de Insight e Diagrama de Afinidades. Os problemas evidenciados, bem como o pensamento empático dos alunos, foram compilados em Cartões de Insight, os quais são reflexões provenientes dos dados das Pesquisas Exploratória, Desk e em Profundidade, transcritas em cartões, contendo um título que resume a informação (tema), o texto original e a fonte (VIANNA et al., 2012). Os Cartões de Insight foram sintetizados em um Diagrama de Afinidades, o qual organiza os cartões de acordo com a similaridade, proximidade e afinidade nas quais cada situação identificada ocorria, delimitando o problema em macro-áreas (VIANNA et al., 2012). Neste projeto, foram consideradas macro-áreas como sendo os ambientes nos quais o problema abordado se desenrolava, facilitando o seu entendimento. Concluindo esta fase, foram elencadas palavras que fazem parte do universo central do problema, as quais permitiram construir uma frase-mãe, que sintetiza a ação central do projeto, para guiar a fase seguinte (VIANNA et al., 2012).

A Fase de Ideação almeja gerar ideias de inovação para solucionar os problemas evidenciados (VIANNA et al., 2012). Para tanto, foi utilizada a ferramenta brainstorming, que consiste em uma técnica que estimula a criação de ideias em um curto período de tempo; quanto mais ideias são geradas, maiores as chances de soluções funcionais e inovadoras (IDEO, 2015; VIANNA et al., 2012). O professor-coordenador estimulou os participantes a exporem suas ideias, atuando como moderador, incentivando a colaboração de todos. As ideias foram combinadas, adaptadas, modificadas e transformadas, num processo de refinamento inicial ao criar conceitos mais complexos para tomada de decisão (IDEO, 2015; VIANNA et al., 2012). 
A Fase de Prototipação tem como função auxiliar a validação das decisões provenientes da fase anterior (DEITTE; OMARY, 2019; VIANNA et al., 2012). Esta fase foi composta por Protótipos em Papel e de Serviços (VIANNA et al., 2012). O protótipo é a tangibilização de uma ideia, a passagem do abstrato para o físico, mesmo que de maneira simplificada (DESIGN COUNCIL, 2015; VIANNA et al., 2012). O Protótipo em Papel consistiu na elaboração de um fluxograma de direcionamento dos pacientes, contendo as ideias para estruturação de um Setor de Triagem integrado ao funcionamento das demais clínicas odontológicas na UEPG. O Protótipo de Serviços é uma simulação da solução proposta (VIANNA et al., 2012). No caso do projeto, foi realizada a simulação do acolhimento, recepção, encaminhamento e rechamada dos pacientes, de acordo com o fluxograma pré-estabelecido.

A Fase de Implementação objetiva executar os processos e serviços testados na fase anterior, podendo ser considerados a melhor solução, ou ser necessário realizar protótipos de outras ideias (DEITTE; OMARY, 2019; DESIGN COUNCIL, 2015; VIANNA et al., 2012). Desta forma, nessa etapa iniciou-se o funcionamento da Recepção com as atividades de acolhimento, cadastro, agendamento, encaminhamento, rechamadas e organização dos atendimentos de urgência. Uma vez finalizados e aprovados, os serviços passam a ser divulgados e utilizados (DESIGN COUNCIL, 2015). Registros, discussões e reflexões em grupo foram realizados em todas as fases.

\section{Resultados}

Utilizando o processo de Design Thinking, foi possível compreender o contexto do problema, gerar ideias solucionadoras, desenvolver protótipos e iniciar o funcionamento das atividades na Recepção das Clínicas Odontológicas, passo inicial para a estruturação do Setor de Triagem Odontológica da UEPG.

Na Fase de Imersão Preliminar, através da Pesquisa Exploratória, observando e interagindo com as pessoas envolvidas no processo, foram identificadas fragilidades no modelo de triagem existente. Segundo as palavras dos acadêmicos extensionistas:

\footnotetext{
“[...] não há um Setor de Triagem organizado; [...] faltam informações sobre a triagem no site da instituição; [...] os pacientes enfrentam dificuldades de localização e acesso às clínicas; [...] não há uma Recepção Odontológica; [...] a triagem é realizada por ordem de chegada ou interesse do acadêmico; [...] os pacientes enfrentam longas filas de espera, não sendo considerada a classificação de risco; [...] há repetição de triagens, prontuários, exames clínicos e complementares em diferentes disciplinas; [...] os pacientes enfrentam dificuldades para conseguir atendimento; [...] não existe uma central de encaminhamento dos pacientes; [...] não há controle dos pacientes que já concluíram o tratamento para realizar acompanhamento."
}

Essa fase permitiu que a equipe do projeto se aproximasse do contexto do problema, facilitando entender o ponto de vista da instituição (perspectiva dos alunos, professores e funcionários) e dos usuários (perspectiva dos pacientes). 
Neste momento, foram identificados os atores-chave e ambientes envolvidos no processo de triagem. Os atores identificados foram: pacientes, alunos, professores e funcionários. Na sequência, fez-se um levantamento para determinação aproximada do número de atores. O número de pacientes atendidos pela graduação, no ano de 2016, foi de 5.079 pessoas. Destes, 204 pacientes foram atendidos pelos alunos do $1^{\circ}$ ano do curso (4\%), 156 pelo $2^{\circ}$ ano $(3 \%), 1.442$ pelo $3^{\circ}$ ano $(28 \%), 1.268$ pelo $4^{\circ}$ ano $(25 \%)$, e 2.009 pelo $5^{\circ}$ ano (40\%). Os alunos matriculados totalizaram 294 pessoas, sendo 64 acadêmicos do $1^{\circ}$ ano, 60 do $2^{\circ}$ ano, 57 do $3^{\circ}$ ano, 58 do $4^{\circ}$ ano, e 55 alunos do $5^{\circ}$ ano. Os professores do departamento de Odontologia totalizaram 56 pessoas, sendo 46 docentes efetivos e 10 colaboradores. Os funcionários do curso de Odontologia totalizaram 19 pessoas, lotados na Central de Material e Esterilização (CME) (21\%), manutenção (16\%), secretaria (11\%), clínicas odontológicas $(31 \%)$, radiologia $(5 \%)$ e laboratório de prótese $(16 \%)$. Os ambientes identificados foram: "porta de entrada", recepção, atendimento, encaminhamento e rechamada. Após a pesquisa de campo, observou-se que os problemas da triagem odontológica não estavam concentrados apenas em um ambiente, havendo falhas desde a "porta de entrada", ou seja, desde a maneira como o paciente chega às clínicas até o momento em que o tratamento é dado como concluído.

Ainda na Fase de Imersão Preliminar, através da Pesquisa Desk, foram identificadas tendências na literatura sobre processos de triagem, como: 1. Classificação de risco, utilizando o protocolo Manchester (AZEREDO et al., 2015; SOUZA; ARAÚJO; CHIANCA, 2015) ou START (Simple Triage and Rapid Treatment), para estratificar a gravidade da condição do paciente (GEBHART; PENCE, 2007); 2. Sistema de etiquetagem, para indicar a prioridade de atendimento (VARSHNEY; MALLOWS; HAMD, 2012); e 3. Avaliação da satisfação dos envolvidos no processo (funcionários e/ou usuários), para conhecer as potencialidades e fragilidades da instituição e dos serviços, a fim de implementar melhorias (VERNERO et al., 2007; RUIZ et al., 1999). Esse momento de Imersão Preliminar buscou informações sobre o assunto do projeto em outras fontes que não os atores, sendo especialmente útil para ajudar a equipe a compreender melhor os limites e as perspectivas do tema em questão (VIANNA et al., 2012).

$\mathrm{Na}$ Fase de Imersão em Profundidade, as entrevistas foram realizadas com pacientes, alunos, professores e funcionários. Dos pacientes que buscaram atendimento odontológico na UEPG em novembro de 2016, foram entrevistadas 99 pessoas. Destes, $46 \%$ avaliaram como muito ruim a acessibilidade para conseguir tratamento odontológico na UEPG, sendo que $80 \%$ relataram alguma insatisfação, tendo como principais queixas a dificuldade e a demora em conseguir atendimento. Foram entrevistados 30 alunos de cada ano $(n=150)$, correspondendo a $51 \%$ do total de acadêmicos. Quando questionados sobre a satisfação frente ao atual processo de triagem, 92\% dos acadêmicos se mostraram insatisfeitos e 97\% relataram que são diretamente prejudicados quando há falta de pacientes nas disciplinas. Dos professores, 15 foram entrevistados, correspondendo a $28 \%$ do total de docentes do departamento; destes, $53 \%$ consideraram o modelo de triagem existente como muito ruim ou ruim, $27 \%$ preferiram não opinar. Foram entrevistados $58 \%$ dos funcionários $(\mathrm{n}=11)$, divididos de forma aleatória entre os servidores que possuem contato direto com os pacientes (Secretaria, Auxiliares das Clínicas 
Odontológicas e Serviço de Radiologia com n=5) e os que não têm contato direto com os mesmos (Central de Material e Esterilização, Manutenção e Técnicos do Laboratório de Prótese com $n=6)$. Os funcionários avaliaram de maneira positiva a eficiência $(45 \%)$ e qualidade $(91 \%)$ do modelo de triagem existente; porém, quando solicitado para descrever o processo de triagem existente, 64\% relataram não acompanhar ou conhecer apenas parcialmente. As entrevistas colaboraram para o entendimento empático do problema, destinando-se à identificação de necessidades e oportunidades para guiar a geração de soluções (VIANNA et al., 2012).

As fases de Imersão Preliminar e Imersão em Profundidade foram fundamentais para tornarem visíveis os atores, os ambientes e os problemas de um processo de triagem não estruturado dentro de uma instituição de ensino.

$\mathrm{Na}$ Fase de Análise e Síntese, os alunos extensionistas escreveram suas percepções dos problemas identificados em Cartões de Insight, totalizando 168 cartões, sendo que, por vezes, as ideias repetiam-se. A Tabela 1 apresenta os dados gerados a partir dos Cartões de Insight, onde $N$ corresponde ao número de repetições da mesma ideia. A utilização dos Cartões de Insights facilitou a rápida consulta e manuseio das informações coletadas nas fases anteriores, que por sua vez foram agrupadas por similaridade em um Diagrama de Afinidades (Figura 1), o qual permitiu visualizar os problemas que ocorrem em cada ambiente, considerando os diferentes atores. Foram utilizadas diferentes cores de notas adesivas para cada ator-chave: paciente (amarelo), aluno (rosa), professor (laranja) e funcionário (azul).

Tabela 1 - Organização dos Cartões de Insight por similaridade das reflexões acerca dos problemas identificados, agrupados de acordo com os atores e ambientes envolvidos ( $\mathrm{N}=$ número de repetições da mesma ideia).

\begin{tabular}{|c|c|c|c|}
\hline Ambiente & Ator & Problema & $\mathrm{N}$ \\
\hline \multirow[b]{5}{*}{ PORTA DE } & \multirow{6}{*}{ Paciente } & Falta informações de como conseguir atendimento. & 6 \\
\hline & & Falta divulgação sobre os tratamentos realizados. & 5 \\
\hline & & Falta divulgação de cronogramas. & 2 \\
\hline & & Falta divulgação sobre o processo de triagem. & 4 \\
\hline & & Dificuldade na localização das clínicas. & 3 \\
\hline \multirow{4}{*}{ ENTRADA } & & Dificuldade de acessibilidade. & 3 \\
\hline & Aluno & $\begin{array}{l}\text { Dificuldade de conseguir pacientes para disciplinas } \\
\text { específicas. }\end{array}$ & 4 \\
\hline & Professor & Dificuldade de conseguir pacientes para pesquisa. & 1 \\
\hline & Funcionário & Falta informações de como ocorre a triagem. & 3 \\
\hline \multirow{5}{*}{ RECEPÇÃO } & Paciente & $\begin{array}{l}\text { Falta de um local para fornecer informações. } \\
\text { Falta de um local para conseguir agendamentos. }\end{array}$ & $\begin{array}{l}7 \\
9 \\
\end{array}$ \\
\hline & \multirow{3}{*}{ Aluno } & Falta de um sistema para buscar por pacientes. & 8 \\
\hline & & Falta de uma central para encaminhamentos. & 5 \\
\hline & & Falta de uma central para organizar rechamadas. & 3 \\
\hline & Professor & Falta de um banco de dados. & 1 \\
\hline
\end{tabular}




\begin{tabular}{|c|c|c|c|}
\hline \multirow{8}{*}{ ATENDIMENTO } & Paciente & $\begin{array}{l}\text { Dificuldade de conseguir atendimento. } \\
\text { Longas filas de espera. } \\
\text { Repetição de exames em cada clínica. } \\
\text { Falta de atendimento integral. }\end{array}$ & $\begin{array}{l}6 \\
5 \\
9 \\
4\end{array}$ \\
\hline & & $\begin{array}{l}\text { Triagem realizada sem considerar a classificação de risco. } \\
\text { Triagem e atendimento realizados de acordo com o } \\
\text { interesse do aluno. }\end{array}$ & $\begin{array}{l}4 \\
3\end{array}$ \\
\hline & Aluno & $\begin{array}{l}\text { Repetição da anamnese, exame físico e exames } \\
\text { complementares em cada clínica. }\end{array}$ & 10 \\
\hline & & Dificuldade de acesso aos pacientes triados. & 4 \\
\hline & & $\begin{array}{l}\text { Dificuldade de acesso aos prontuários gerados em outras } \\
\text { disciplinas. }\end{array}$ & 5 \\
\hline & & Extravio de prontuários pelos alunos. & 1 \\
\hline & Professor & Falta de um prontuário único. & 8 \\
\hline & Funcionário & Dificuldade de controle dos prontuários. & 6 \\
\hline \multirow{5}{*}{ ENCAMINHAMENTO } & & Falta de orientações após a triagem. & 3 \\
\hline & Paciente & Falta de orientações após o atendimento clínico. & 4 \\
\hline & & Falta previsão de rechamada para atendimento. & 3 \\
\hline & Aluno & $\begin{array}{l}\text { Dificuldade em conseguir encaminhar os pacientes para } \\
\text { outras disciplinas. }\end{array}$ & 10 \\
\hline & Funcionário & $\begin{array}{l}\text { Falta de um funcionário específico para realizar o } \\
\text { encaminhamento dos pacientes. }\end{array}$ & 1 \\
\hline \multirow{4}{*}{ RECHAMADA } & Paciente & Falta de acompanhamento adequado. & 4 \\
\hline & Aluno & $\begin{array}{l}\text { Dificuldade de acesso aos prontuários arquivados. } \\
\text { Dificuldade de contato com o paciente. }\end{array}$ & $\begin{array}{l}5 \\
4\end{array}$ \\
\hline & Professor & Falta de um controle de rechamadas. & 2 \\
\hline & Funcionário & $\begin{array}{l}\text { Dificuldade de armazenamento, organização e controle } \\
\text { dos prontuários arquivados. }\end{array}$ & 3 \\
\hline
\end{tabular}

Fonte: Elaborado pelos autores.

As principais defasagens identificadas foram: a dificuldade dos pacientes para conseguir atendimento; a falta de informações sobre o processo de atendimento odontológico; a inexistência de aspectos de funcionamento básicos, como acolhimento, agendamentos, encaminhamentos e rechamadas; problemas relacionados aos prontuários, como a dificuldade de acesso, armazenamento e controle; e a repetição de exames (anamnese, exame físico e exames complementares) em cada disciplina. 
Figura 1 - Diagrama de Afinidades, elaborado a partir dos Cartões de Insight, evidenciando os problemas do Setor de Triagem em cada ambiente (cores diferentes representam diferentes atores).

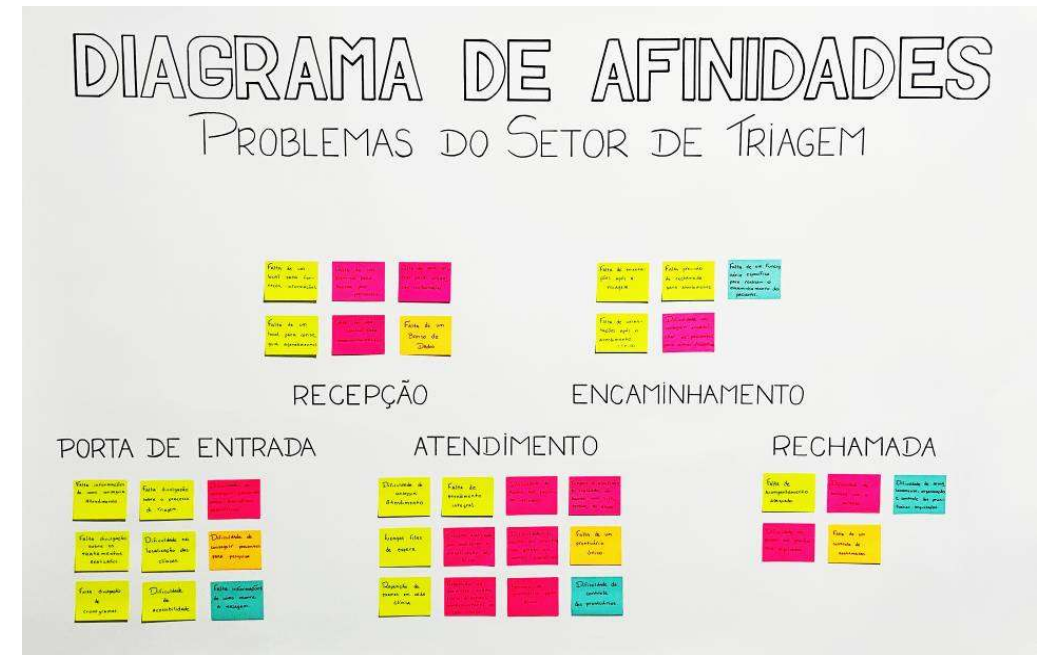

Fonte: Acervo do projeto.

Após a confecção do Diagrama de Afinidades, foram elencadas como palavras que fazem parte do universo central do problema: triagem, pacientes, alunos, professores, funcionários e estruturação. Na sequência, foi construída uma frase-mãe que sintetizava a ação central do projeto: "Como podemos repensar o processo de triagem na UEPG, melhorando a experiência dos pacientes, alunos, professores e funcionários?". Essa fase proporcionou a identificação de padrões e inter-relações das informações e o resultado final auxiliou na compreensão dos dados coletados em campo (VIANNA et al., 2012).

Na Fase de Ideação, a frase-mãe foi utilizada para geração de ideias de possíveis soluções criativas para os problemas evidenciados. A geração de ideias foi realizada através de brainstorming, em reunião com todas as equipes do projeto. Ao utilizar o brainstorming, é importante que haja variedade de perfis de pessoas envolvidas no processo, dessa forma, a heterogeneidade nas equipes de trabalho (alunos de diferentes anos da graduação e pósgraduação) estimulou a discussão e a proposição de soluções através de diferentes óticas, permitindo obter grande diversidade de opiniões, o que é desejável dentro do processo de inovação (VIANNA et al., 2012). Após alguns refinamentos, as ideias de soluções que foram escolhidas para iniciar as ações do projeto foram: 1. Estabelecimento de um fluxo de direcionamento do paciente desde o acolhimento até o final do tratamento; 2. Recepção (espaço físico) como local de referência para proporcionar informações e orientações; 3 . Centralização das ações do Setor de Triagem (acolhimento, agendamento, encaminhamento e rechamada) na Recepção; 4. Organização da sala de arquivos; e 5. Implementação do prontuário odontológico único.

Na Fase de Prototipação, através de Protótipo em Papel, foi criado um fluxograma de direcionamento (Figura 2), levando em consideração todos os ambientes pelos quais o 
paciente passa até conseguir ter o seu tratamento concluído, prevendo encaminhamentos internos ou externos. O fluxograma tem por objetivo guiar os atores através das diversas etapas de atendimento.

Figura 2 - Fluxograma de atendimento odontológico na UEPG criado na Fase de Prototipação para orientar os atores desde o acolhimento do paciente até a conclusão do tratamento.

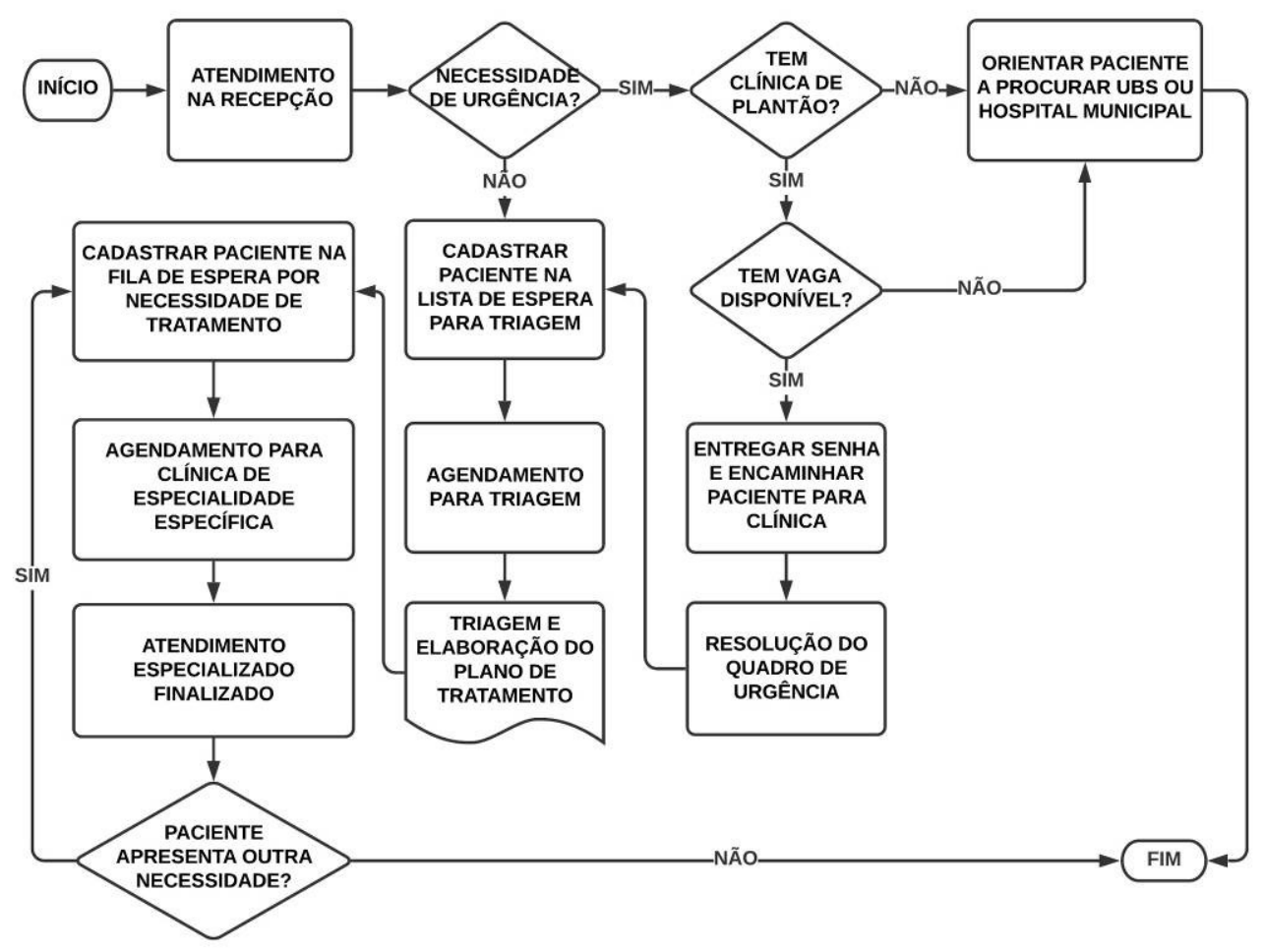

Fonte: Elaborado pelos autores.

Na sequência, através de Protótipo de Serviços, foram realizados treinamentos e capacitações dos extensionistas, culminando com a simulação das atividades (acolhimento, agendamento, encaminhamento e rechamada) que seriam desenvolvidas na Recepção. A Fase de Prototipação permitiu que as ideias de solução, através de simulações rápidas e de instrumentos de baixo custo, fossem refinadas, baseadas no feedback dos envolvidos, antes de ter sido investido muito tempo e/ou recursos (DEITTE; OMARY, 2019). Após a etapa de simulação, foi verificada a viabilidade das soluções propostas e as mesmas foram validadas. Este processo foi importante, pois auxiliou no entendimento do que se pretende implementar, facilitando a identificação de uma solução final funcional (VIANNA et al., 2012).

Na Fase de Implementação, realizou-se a organização e limpeza da sala de arquivos onde armazenam-se os prontuários físicos dos pacientes atendidos nos anos anteriores, com 
o objetivo de, posteriormente, realizar a digitalização dos mesmos. Durante esta etapa, iniciouse o funcionamento das atividades na Recepção. A Recepção das Clínicas Odontológicas foi inaugurada no dia 11 de agosto de 2017 pelo Setor de Ciências Biológicas e da Saúde (Sebisa), a partir da aplicação de recursos obtidos através do curso de especialização em Ortodontia (lato sensu) (Figuras 3 e 4). As atividades iniciaram com caráter informativo, além da realização de cadastros, utilizando o Microsoft Office Excel (Microsoft $\AA$ ); agendamentos para efetuar a triagem; encaminhamentos e rechamada para atendimento clínico; e organização dos atendimentos de urgência (plantões), levando em consideração a classificação de risco. A Recepção funciona 40 horas semanais, com todas as atividades sendo realizadas pelos alunos extensionistas, sob supervisão de um funcionário do departamento e coordenação dos alunos de pós-graduação e professores integrantes do projeto. Desde a inauguração até o final do ano de 2018, foram acolhidas 2.006 pessoas na Recepção.

Figura 3 - Construção do espaço físico da Recepção, localizada junto às Clínicas Odontológicas no bloco $\mathrm{M}$ do Campus de Uvaranas.

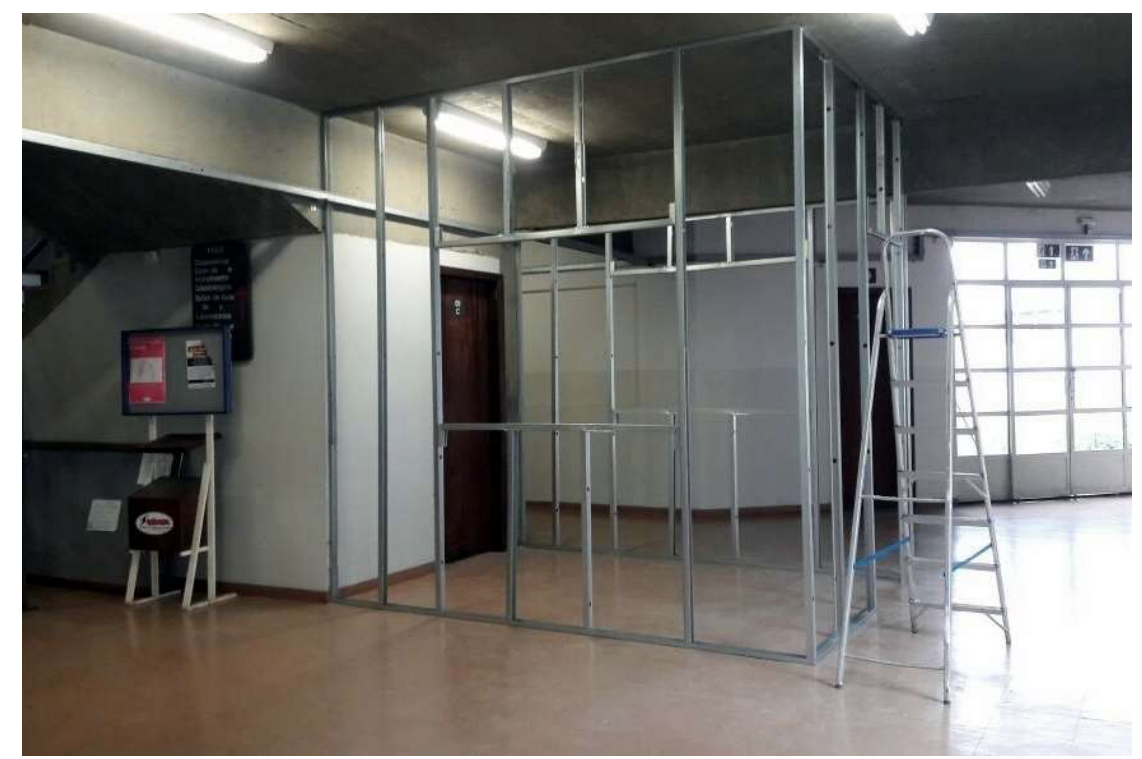

Fonte: Acervo do projeto. 
Figura 4 - Recepção das Clínicas Odontológicas da UEPG, local de referência para proporcionar informações e de centralização das ações do Setor de Triagem (acolhimento, agendamento, encaminhamento e rechamada).

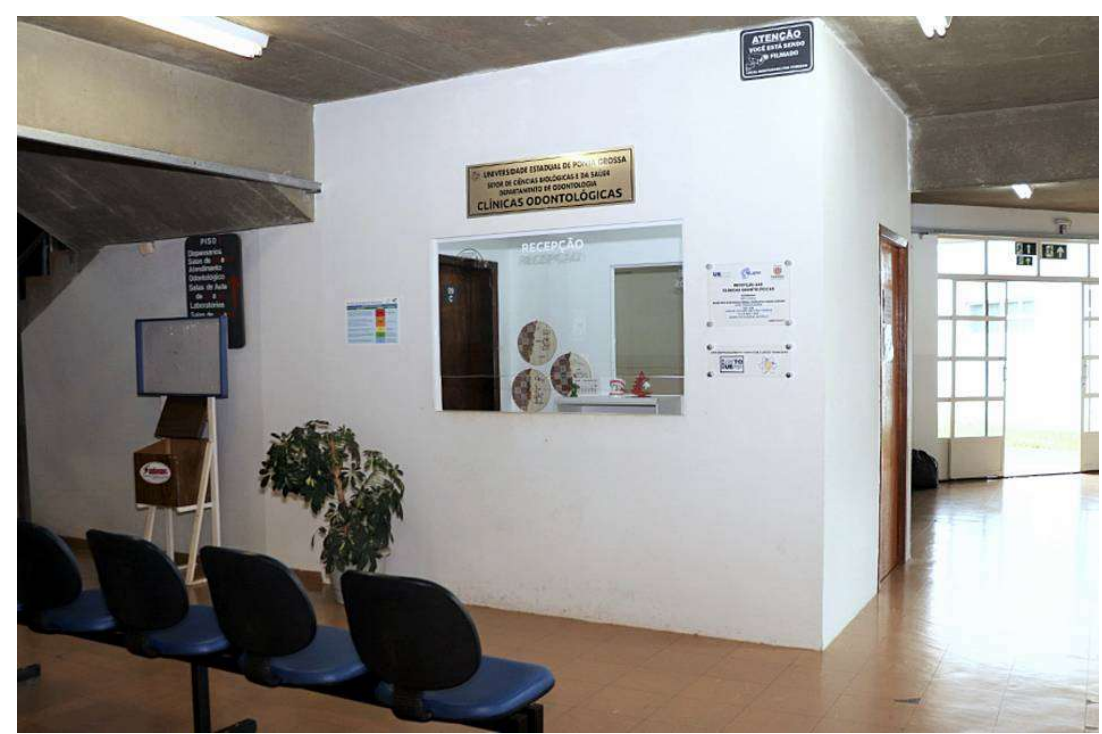

Fonte: Acervo do projeto.

As Fases de Prototipação e Implementação foram concomitantes durante o processo, pois não se trata de um método perfeitamente linear (IDEO, 2015). Durante todas as fases, buscou-se colocar o ser humano no centro do processo, tanto para entender o usuário final (paciente) quanto para envolver os colaboradores constantes (alunos, professores e funcionários) (VIANNA et al., 2012).

Apesar do dinamismo do processo Design Thinking, a implementação rápida de um sistema completamente novo de triagem é inviável. O processo envolve uma grande variedade de atores com subjetividades (conhecimentos, habilidades, perspectivas, costumes, objetivos e culturas de trabalho) que podem dificultar a instalação de processos de inovação e mudança (JANSSEN; VLIERT; WEST, 2004). A implementação de um adequado Setor de Triagem necessita de infraestrutura, com espaços projetados para o desenvolvimento de ações inerentes ao processo, assim como recursos humanos capacitados, estando estas limitações diretamente relacionadas à disponibilidade de recursos financeiros. Dentre as atividades que ainda não foram concluídas estão a digitalização dos prontuários físicos antigos e implementação de um prontuário único. Estão sendo conduzidos pilotos em duas clínicas da graduação (Periodontia e Clínica Integrada I), com atendimento utilizando o sistema de prontuário único físico, o qual, futuramente, pretende-se expandir a todas as disciplinas.

Embora existam dificuldades em se implementar um projeto de extensão com caráter de inovação, a maior contribuição deste projeto está relacionada com a promoção de melhorias para a sociedade local. A partir dos resultados obtidos pelas ações extensionistas, o paciente, ao buscar atendimento nas clínicas odontológicas de ensino da UEPG, encontrará: 1) uma "porta de 
entrada" organizada, com melhora do acolhimento, 2) uma Recepção, funcionando como um centro de referência para proporcionar informações e solucionar dúvidas, e 3) um fluxo estabelecido de direcionamento dos pacientes pelas diversas fases do tratamento odontológico (acolhida, agendamento, triagem, encaminhamento, atendimento e rechamadas, até conduzilos à conclusão do tratamento). Além das melhorias para a comunidade, o projeto traz como benefício a formação diferenciada dos alunos extensionistas. O envolvimento dos alunos da graduação na construção de mudanças contribui para a formação de competências e habilidades, indo ao encontro das Diretrizes Curriculares Nacionais (DCN) dos cursos de Odontologia (BRASIL, 2002), promovendo o amadurecimento crítico e humanitário desses alunos (MOIMAZ et al., 2015).

As DCN preconizam uma nova visão do atendimento, de forma a garantir a integralidade da assistência (BRASIL, 2002). No modelo antigo, a produtividade (estabelecimento de uma produção mínima) era o foco principal, ao passo que, no novo modelo, a ênfase incide sobre o desenvolvimento do diagnóstico e planejamento, com uma abordagem integral do paciente (FERRETTI et al., 2012). Nesse contexto, o Setor de Triagem vem a contribuir diretamente nestas etapas, através do acolhimento e avaliação inicial das necessidades, e proporciona o acompanhamento do paciente por todo o processo, garantindo o acesso e encaminhamentos necessários para a abordagem integral à saúde. A partir das atividades iniciais do projeto, foi possível verificar os próximos desafios. A continuidade do projeto pretende substituir o prontuário único físico por um prontuário único eletrônico e o uso do Microsoft Office Excel (Microsoft ${ }^{\circledR}$ ) por um software de gerenciamento, permitindo realizar os cadastros, agendamentos e encaminhamentos de maneira mais eficiente.

Devido à grande demanda de pacientes que buscam por atendimento na UEPG, verificou-se a necessidade de instituir uma clínica específica para triagem e também para atendimento de urgências, as quais serão planejadas na sequência. Assim, muitos problemas ainda persistem, pois a implementação de mudanças demanda tempo e passa por dificuldades até que as melhorias alcançadas sejam consolidadas.

\section{Conclusões}

O projeto de extensão universitária apresentado neste artigo objetiva estruturar um Setor de Triagem considerando a necessidade de readequar o funcionamento da triagem odontológica na UEPG, visando melhorar o atendimento à comunidade. Este projeto pode ser levado como um exemplo de processo de inovação aplicado à extensão universitária, na área da saúde. A metodologia utilizada permitiu compreender o problema, propor ideias de solução, testar protótipos e implementar mudanças.

Porém, a estruturação do Setor de Triagem Odontológica da UEPG está longe de ser finalizada. Futuramente, como medida solucionadora dos problemas remanescentes, objetivase implementar um prontuário único eletrônico, um software de gerenciamento, uma clínica 
exclusiva de triagem e de atendimento de urgências. Entretanto, as ações já realizadas viabilizarão melhorias na qualidade dos serviços ofertados para a comunidade, uma vez que o fluxo de direcionamento dos pacientes se encontra estruturado, desde a "porta de entrada" até as rechamadas, sendo os pacientes adequadamente acolhidos e direcionados para o atendimento integral de suas necessidades. Serão realizados estudos para avaliar as alterações realizadas, buscando o aprimoramento constante do processo.

\section{Referências}

AZEREDO, T. R. et al. Efficacy of the Manchester Triage System: a systematic review. International Emergency Nursing, v. 23, n. 2, p. 47-52, abr. 2015.

BRASIL. Ministério da Educação. Conselho Nacional da Educação. Câmara da Educação Superior. Resolução CNE/CES 3/2002: Instituiu as Diretrizes Curriculares Nacionais do Curso de Graduação em Odontologia. Brasília, 2002. 10 p.

DEITTE, L. A.; OMARY, R. A. The Power of Design Thinking in Medical Education. Academic Radiology, v. 26, n. 10, p. 1417-1420, out. 2019.

DESIGN COUNCIL (org.). Design methods for developing services: An introduction to service design and a selection of service design tools. Londres, 2015. 23 p.

DOCHERTY, C. Perspectives on Design Thinking for Social Innovation. Design Journal, v. 20, n. 6, p. 719-724, out. 2017.

EBERHART, A. et al. Using a human-centered design approach for collaborative decisionmaking in pediatric asthma care. Public Health, v. 170, p. 129-132, maio 2019.

FERREIRA, F. K. et al. New mindset in scientific method in the health field: Design Thinking. Clinics, v. 70, n. 12, p. 770-772, dez. 2015.

FERRETTI, L. H. et al. Avaliação discente e as Diretrizes Curriculares Nacionais - realidade das clínicas integradas da UNIVILLE. Revista da ABENO, v. 12, n. 2, p. 155-62, 2012.

GEBHART, M. E.; PENCE, R. START triage: does it work? Disaster Management \& Response, v. 5, n. 3, p. 68-73, jul./set. 2007.

GONÇALVES, E. R.; VERDI, M. I. M. A vulnerabilidade e o paciente da clínica odontológica de ensino. Revista Brasileira de Bioética, v. 1, n. 2, p. 195-205, 2005.

GONÇALVES, E. R.; VERDI, M. I. M. Os problemas éticos no atendimento a pacientes na clínica odontológica de ensino. Ciência \& Saúde Coletiva, v. 12, n. 3, p. 755-764, 2007. 
IDEO (org.). The Field Guide to Human-Centered Design. 1. ed. Canadá: Design Kit, 2015. 192 p.

INSTITUTO BRASILEIRO DE GEOGRAFIA E ESTATÍSTICA (IBGE). Pesquisa Nacional de Saúde 2013: acesso e utilização dos serviços de saúde, acidentes e violências Brasil, Grandes Regiões e Unidades da Federação. Rio de Janeiro: Instituto Brasileiro de Geografia e Estatística, 2015. 100 p.

JANSSEN, O; VLIERT, E. van de; WEST, M. The bright and dark sides of individual and group innovation: a Special Issue introduction. Journal of Organizational Behavior, v. 25, n. 2, p. 129-145, fev. 2004.

LISLE, A. H.; MERENDA, C.; GABBARD, J. Using affinity diagramming to generate a codebook: a case study on young military veterans and community reintegration.

Qualitative Research, p. 1-18, jun. 2019.

LISTL, S. et al. Global Economic Impact of Dental Diseases. Journal of Dental Research, v. 94, n. 10, p. 1355-1361, out. 2015.

MOIMAZ, S. A. S. et al. Extensão universitária na ótica de acadêmicos: o agente fomentador das Diretrizes Curriculares Nacionais. Revista da ABENO, v. 15, n. 4, p. 45-54, 2015.

PETERSEN, P. E. The World Oral Health Report 2003: continuous improvement of oral health in the 21 st century - the approach of the WHO Global Oral Health Programme. Community Dentistry and Oral Epidemiology, v. 31, n. 1, p. 3-23, dez. 2003.

ROBERTS, J. P. et al. A design thinking framework for healthcare management and innovation. Healthcare, v. 4, n. 1, p. 11-14, mar. 2016.

RUIZ, U. et al. A two-level integrated approach to self-assessment in healthcare organizations. International Journal of Health Care Quality Assurance, v. 12, n. 4, p. 135-142, 1999.

SOUZA, C. C.; ARAÚJO, F. A.; CHIANCA, T. C. Scientific Literature on the Reliability and Validity of the Manchester Triage System (MTS) Protocol: A Integrative Literature Review. Revista da Escola de Enfermagem da USP, v. 49, n. 1, p.144-151, fev. 2015.

VARSHNEY, K.; MALLOWS, J.; HAMD, M. Disaster triage tags: Is one better than another? Emergency Medicine Australasia, v. 24, n. 2, p.187-193, abr. 2012.

VERNERO, S. et al. A two-level EFQM self-assessment in an Italian hospital. International Journal of Health Care Quality Assurance, v. 20, n. 3, p. 215-231, 2007.

VIANNA, M. et al. Design thinking: inovação em negócios. Rio de Janeiro: MJV Press, 2012. 162 p. 